\title{
5 Years of Experience Implementing a Methicillin-Resistant Staphylococcus aureus Search and Destroy Policy at the Largest University Medical Center in the Netherlands
}

\author{
Margreet C. Vos, MD, PhD; Myra D. Behrendt, MSc; Damian C. Melles, MD; Femke P. N. Mollema, MSc; \\ Woutrinus de Groot, RN; Gerard Parlevliet, RN; Alewijn Ott, MD, PhD; Deborah Horst-Kreft, LT; \\ Alex van Belkum, PhD; Henri A. Verbrugh, MD, PhD
}

ов јестіve. To evaluate the effectiveness of a rigorous search and destroy policy for controlling methicillin-resistant Staphylococcus aureus (MRSA) infection or colonization.

DESIGN. Hospital-based observational follow-up study.

Setting. Erasmus University Medical Center Rotterdam, a 1,200-bed tertiary care center in Rotterdam, the Netherlands.

METHODs. Outbreak control was accomplished by the use of active surveillance cultures for persons at risk, by the preemptive isolation of patients at risk, and by the strict isolation of known MRSA carriers and the eradication of MRSA carriage. For unexpected cases of MRSA colonization or infection, patients placed in strict isolation or contact isolation and healthcare workers (HCWs) were screened. We collected data from 2000-2004.

RESUlts. During the 5-year study period, 51,907 MRSA screening cultures were performed for 21,598 persons at risk (8,403 patients and 13,195 HCWs). By screening, it was determined that $123(1.5 \%)$ of 8,403 patients and $31(0.2 \%)$ of 13,195 HCWs were MRSA carriers. From the performance of clinical cultures, it was determined that 54 additional patients were MRSA carriers, resulting in a total of 177 patients carrying MRSA. Of the 177 patients carrying MRSA, 144 (81\%) were primary patients, and 33 (19\%) secondary patients. The average number of nosocomial transmissions was 6.7 per year. The cumulative incidence of MRSA colonization among this group of patients was 0.10 cases per 100 admissions. Of 156 cases of MRSA colonization, 44 (28\%) were acquired in a foreign healthcare institution, and $45(29 \%)$ were acquired in other Dutch hospitals, $22(47 \%)$ of which were acquired in a single hospital in our region. There were 16 cases $(10 \%)$ that occurred in a nursing home and another 16 cases $(10 \%)$ that fulfilled our definition of community-acquired MRSA colonization; there were 4 cases $(3 \%)$ categorized as "other" and 31 cases $(20 \%)$ for which the source of MRSA acquisition remained unknown. The basic reproduction rate was 10 -fold less for patients isolated on admission, compared with those who were not. During the 5 -year study period, 5 episodes of MRSA bacteremia occurred in which 4 patients died, an incidence rate of 0.28 cases of infection per 100,000 patient-days per year.

CONCLUSION. Our results show that, during a rigorous search and destroy policy, a low incidence of MRSA in our medical center was continuously observed and that this policy most likely contributed to a very low nosocomial transmission rate.

Infect Control Hosp Epidemiol 2009; 30:977-984

Methicillin-resistant Staphylococcus aureus (MRSA) is an important cause of nosocomial and community-acquired infections worldwide. In many countries, the percentage of MRSA isolates recovered from patients in medical or nursing centers has now reached $20 \%-50 \%$ of all clinical isolates of S. aureus. The Netherlands and the Nordic European countries have so far succeeded in keeping the incidence of MRSA infection or colonization low (approximately $1 \%$ ). ${ }^{1}$ The secret of this success is thought to be a combination of both restrictions on the prescription of antibiotics and implemen- tation of a strict national prevention policy. ${ }^{2}$ In the Netherlands, antibiotic pressure is low, compared with other countries, ${ }^{3}$ and a search and destroy policy for the prevention of MRSA infection or colonization was elaborated as a Dutch national strategy by the Working Party on Infection Prevention and has been in place since $1988 .^{2}$ The measures described in the policy were implemented in addition to the universal standard precautions. The MRSA search and destroy policy focuses on (1) defining groups at risk and screening of both patients and healthcare workers (HCWs) at risk, (2)

From the Department of Medical Microbiology and Infectious Diseases, Erasmus University Medical Center Rotterdam, Rotterdam, the Netherlands (all authors).

Received January 6, 2009; accepted April 28, 2009; electronically published August 27, 2009.

(C) 2009 by The Society for Healthcare Epidemiology of America. All rights reserved. 0899-823X/2009/3010-0008\$15.00. DOI: 10.1086/605921 
TABLE 1. Risk Categories for Methicillin-Resistant Staphylococcus aureus (MRSA) Carriage

\begin{tabular}{|c|c|c|}
\hline Risk category & Patients & Healthcare workers \\
\hline Category $1^{\text {a }}$ & Culture result positive for MRSA & Culture result positive for MRSA \\
\hline Category $2^{\mathrm{b}}$ & $\begin{array}{l}\text { Treated in foreign healthcare institution; transferred from } \\
\text { medical center with an outbreak of MRSA not brought } \\
\text { under control; had contact with an individual with } \\
\text { proven MRSA colonization or infection; an adopted } \\
\text { child from abroad }\end{array}$ & $\begin{array}{l}\text { Unintentional unprotected contact with MRSA carrier; } \\
\text { treated or worked in foreign healthcare institution }\end{array}$ \\
\hline Category $3^{c}$ & Not belonging to category 1 or 2 & Not belonging to category 1 or 2 \\
\hline
\end{tabular}

a Proven carriers of MRSA.

${ }^{\mathrm{b}}$ High risk of carrying MRSA.

${ }^{c}$ No increased risk.

strict isolation of MRSA-positive patients and of patients considered to be at risk pending culture results, (3) outbreak management, and, after hospital discharge, (4) follow-up of carriers and (5) elimination of carriage if feasible. In our study, we present the outcome associated with the search and destroy policy in our hospital. We studied the effect of infection control and outbreak management on incidence rates for groups at risk and not at risk and on transmission rates of MRSA, and we determined the sources and diversity of MRSA strains.

\section{METHODS}

\section{Setting, Design, and Participants}

The Erasmus University Medical Center Rotterdam (hereafter referred to as Erasmus MC) is a 1,200-bed tertiary care center in Rotterdam, the Netherlands, with approximately 35,000 admissions, 311,000 patient-days, and 497,000 outpatient visits yearly. The measures in the search and destroy policy used by Erasmus MC are based on the national policy for MRSA from the Dutch Working Party on Infection Prevention (2000-2004). ${ }^{2}$

The present study is a hospital-based observational cohort study. We measured the cumulative incidence of MRSA infection or colonization among patients and HCWs deemed at risk according to criteria from the search and destroy policy and determined the number of persons who were infected or colonized with MRSA during their stay in the hospital. The sources of MRSA infection or colonization, the genotypes of MRSA, and the preventive effect of preemptive isolation on transmission rates were determined. All of the patients who were admitted to the Erasmus MC during the period from 2000 to 2004 and all of the HCWs who were involved in their care were categorized into risk groups defined in the search and destroy policy (Table 1).

\section{Definitions}

Primary cases were individuals with proven MRSA carriage who acquired MRSA in either foreign healthcare institutions, other Dutch healthcare institutions, or the community. It was determined, either epidemiologically or by molecular typing of their strain, that they did not become carriers via the transmission of MRSA in our center. MRSA was considered to be acquired in the community if the carrier, in the year before detection, had not been admitted to or treated in any healthcare facility. Secondary cases were individuals who were infected or colonized as a result of the transmission of an MRSA strain in the Erasmus MC as determined by epidemiological and molecular typing data, who were admitted to the same ward during the same period as the primary patient (ie, the index patient), and who shared the same strain as the primary patient.

The source of MRSA carriage was considered to be unknown if, in the past, the patient had been admitted to a healthcare center in the Netherlands that did not report an outbreak or a single case of MRSA infection or colonization with the same pulsed-field gel electrophoresis (PFGE) type. Individual healthcare centers other than Erasmus MC were actively approached for documentation of the emergence of defined PFGE types and outbreaks of MRSA infection in their healthcare facilities.

\section{Screening Cultures}

Samples for active screening culture were obtained from risk category 2 patients and HCWs. For patients, these samples were obtained from the nose, throat, perineum, nonintact skin, urine (in the case of urinary catheter use), drain fluid (in the case of drain use), and/or exit sites (in the case of drain and/or catheter use). The screening cultures of a single person (case or HCW) are defined as a culture set. Isolation of patients suspected of MRSA carriage was discontinued when the culture set tested negative for MRSA. For HCWs, samples for culture were obtained from the nose, throat, and, if present, any skin lesions. This testing of samples obtained from HCWs for culture does not require informed consent, because this type of testing is part of the hospital MRSA search and destroy policy, which is explained at the start of employment.

\section{Preventive Measures}

From hospital admission onward, patients who were categorized as known carriers of MRSA (ie, category 1) or as being at increased risk of carrying MRSA (ie, category 2) were cared for in strict isolation in a single room with closed door, preferably with an anteroom and regulated negative air 


\section{A. Working scheme after unexpected detection of MRSA in nonisolated patient (index patient):}

\author{
1. Index patient moves from category 3 to \\ category 1 : \\ This means: \\ $\rightarrow$ Put into strict isolation, door closed, \\ desinfection daily \\ $\rightarrow$ Labeled as MRSA patient in the \\ electronic hospital system
}

2. Patients cared for in the same room as the index patient move from category 3 to category 2:

This means:

$\rightarrow$ Put into cohort strict isolation

$\rightarrow$ Samples obtained from all body sites for screening cultures

\section{All patients admitted to the same department during the same admission period (regardless of their place of stay) move from category 3 to category 2 : This means: \\ $\rightarrow$ Samples obtained from all body sites for screening cultures \\ $\rightarrow$ No isolation}

4. HCWs having had direct or indirect contact with the index patient move from category 3 to category 2 :

This means:

$\rightarrow$ Samples obtained from nose and throat for screening cultures

\section{B. Working scheme after detection of MRSA in one or more contact persons, either HCW or patient:}

\author{
One of the contact persons positive: \\ Assumption that transmission had occurred: \\ outbreak ongoing: \\ This means: \\ $\rightarrow$ Ward closed for new admissions \\ $\rightarrow$ All contact patients are preemptively \\ placed in strict isolation and again screened, \\ regardless of their location of stay \\ $\rightarrow$ All HCWs of the department are \\ screened again \\ $\rightarrow$ Personnel of the "closed" ward are \\ not allowed to leave the ward unless \\ wearing protective clothing (mask/gown/ \\ gloves/cap) (for protection of individuals \\ outside of ward against contamination with \\ MRSA) \\ $\rightarrow$ HCWs from other wards are only \\ allowed to enter the "closed" ward \\ wearing gowns, gloves, mask, and cap. \\ (to prevent them from acquiring MRSA). \\ $\rightarrow$ Visitors must wear gowns and \\ wash hands. They are not allowed to \\ visit another patient immediately after \\ visiting the MRSA patient. \\ $\rightarrow$ If an HCW was found to carry MRSA, \\ he or she is immediately furloughed from \\ direct patient care. \\ $\rightarrow$ All floors of the ward are disinfected \\ twice daily (chlorine $250 \mathrm{ppm}$ ). \\ $\rightarrow$ New screening rounds are initiated \\ after each new positive finding \\ $\rightarrow$ When no new carriers are identified, \\ the ward will be completely disinfected. \\ $\rightarrow$ Items that cannot be disinfected are \\ discarded.
}

FIGURE 1. Working scheme after unexpected detection of methicillin-resistant Staphylococcus aureus (MRSA) in nonisolated patients (A) and after finding a secondary case $(B)$. HCW, healthcare worker.

pressure in the patient room and anteroom. The attending HCWs wore masks, gowns, gloves, and caps upon entering the isolation room. Thorough disinfection of the hands with alcohol-based hand rub was required after removing the gloves, gown, mask, and cap. The room was disinfected twice daily (chlorine $250 \mathrm{ppm}$ ). After an MRSA carrier was discharged from the hospital, the isolation room was thoroughly disinfected; all objects that could not be disinfected were discarded. Patients were followed up after hospital discharge and received decolonization treatment as soon as catheters, drains, and/or wounds were no longer present. In short, decolonization was defined as a mupirocin and chlorhexidin body wash; in case of extra nasal site colonization, 2 oral antibiotics were also given. After decolonization treatment, 6 consecutive culture sets had to test negative for MRSA before the patient could be considered free of MRSA colonization. Up to that time, the patient remained in category 2, which meant that strict isolation upon readmission to the hospital or during outpatient clinic visits was required (see below).
HCWs who were known carriers of MRSA (ie, category 1) were immediately furloughed from patient care and received MRSA eradication treatment at once. They remained furloughed until the first screening samples for culture, obtained 3 days after the end of eradication treatment, were found to be negative. Thereafter, HCWs were tested for MRSA by culture once weekly. After 6 culture sets tested negative for MRSA, the HCW was considered free of MRSA colonization, and follow-up was discontinued. HCWs at increased risk of MRSA carriage (ie, category 2) were allowed to work without preventive measures but were screened once (eg, after returning from work in a foreign healthcare institution) or as long as the risk factor(s) remained present (Table 1).

\section{Unexpected MRSA and Outbreak Management}

Clinical culture samples unexpectedly yielding MRSA immediately led to the initiation of a bundle of prevention and screening measures if the patient (ie, the index patient) was 


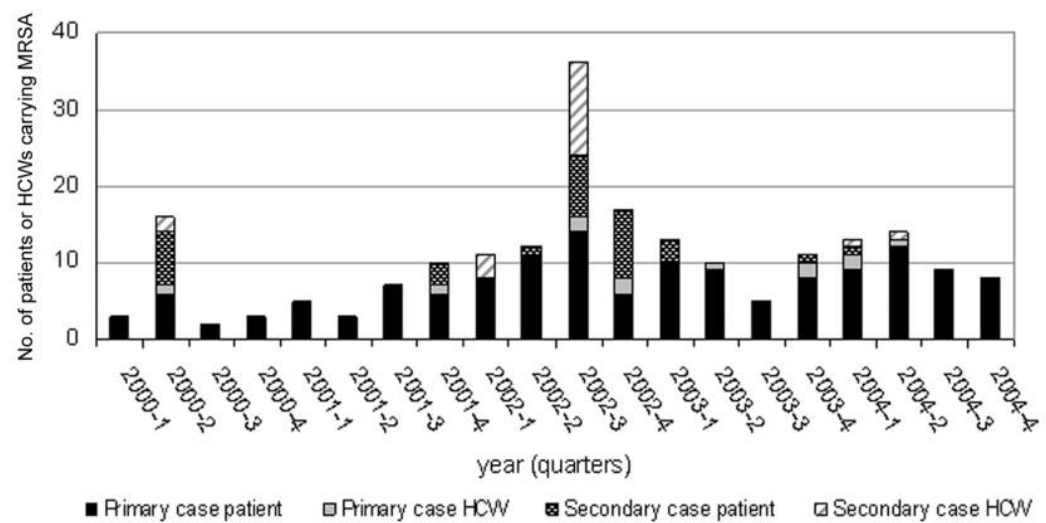

FIGURE 2. New findings of methicillin-resistant Staphylococcus aureus (MRSA) carriage among patients and healthcare workers (HCWs) in Erasmus University Medical Center Rotterdam, Rotterdam, the Netherlands, during 2000-2004, per quarter. There were 177 patients and $31 \mathrm{HCWs}$ who tested positive for carrying MRSA. There were 144 (81\%) of 177 patients and 12 (39\%) of $31 \mathrm{HCWs}$ who were considered primary cases (ie, individuals with proven MRSA carriage who acquired MRSA in either foreign healthcare institutions, other Dutch healthcare institutions, or the community). There were 33 (19\%) of 177 patients and 19 (61\%) of 31 HCWs who were considered secondary cases (ie, individuals who were infected or colonized as a result of the transmission of an MRSA strain in our medical center as determined by epidemiological and molecular typing data, who were admitted to the same ward during the same period as the primary patient, and who shared the same strain as the primary patient).

not already in strict isolation. The measures used after a secondary patient is found to be a carrier of MRSA are described in Figure 1.

\section{Data Collection}

Data were collected from reports of the infection control practitioners, laboratory reports, electronic charts, and medical charts and by interviewing the staff on the wards with MRSA carriers. The following data were systematically collected: the number of screening cultures, their test results, the source of MRSA for each new case, whether the case was primary or secondary, whether preemptive isolation was initiated at admission for patients found to be in category 1 or category 2, the number of wards closed, and the duration of closure.

\section{Microbiological Methods}

MRSA screening swabs were first inoculated onto blood agar plates (Becton Dickinson) and thereafter put into phenol red mannitol enrichment broth containing $5 \mathrm{mg} / \mathrm{L}$ ceftizoxime and $75 \mathrm{mg} / \mathrm{L}$ aztreonam. ${ }^{4}$ After $24 \mathrm{hr}$ incubation at $35^{\circ} \mathrm{C}$, the blood agar plates were checked for the growth of $\geqslant 15$ colonyforming units of any bacterial species. If fewer colony-forming units were present, the sampling was deemed to have been insufficient, and a new swab was requested. After 48 hours of incubation, 1 loop $(1 \mu \mathrm{L})$ of the broth was subcultured onto a blood agar plate. Microbiological methods to identify and type MRSA are described elsewhere. ${ }^{5}$

\section{Data Analysis}

Statistical analysis of the data was performed with Epi Info software, version 2002 (Centers for Disease Control and Pre- vention). Differences in frequencies were evaluated by use of a $\chi^{2}$ test. Basic reproduction rates were calculated. A $P$ value of less than .05 was considered to be statistically significant. The $95 \%$ confidence intervals and the $P$ values for differences between groups were based on an exact Poisson test.

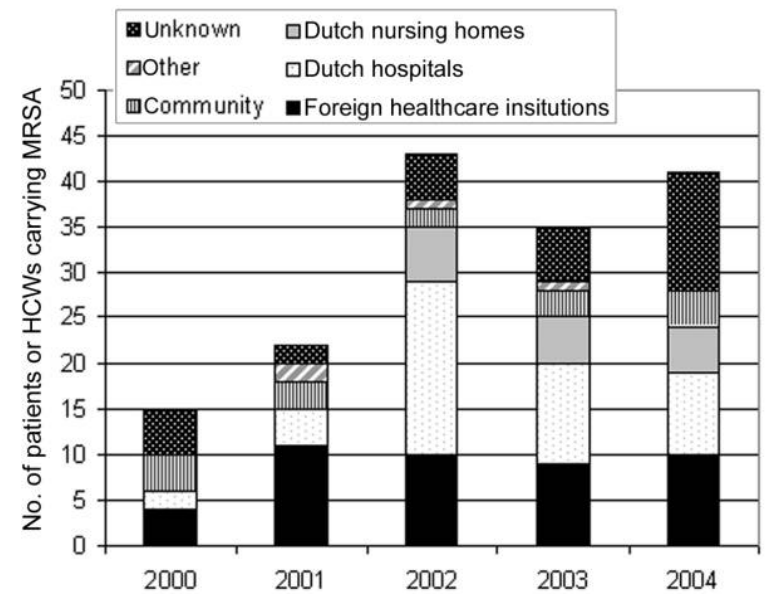

FIGURE 3. Origin of methicillin-resistant Staphylococcus aureus (MRSA) acquisition for 144 primary patients and 12 primary HCWs, resulting in a total of 156 primary cases. Except for all cases occurring in "Foreign healthcare institutions" and except for some cases occurring in other "Dutch hospitals," the sources of MRSA are not proven, but the individuals are assumed to be at risk for acquiring MRSA. "Foreign healthcare institutions" include both long-term care and medical centers in foreign countries, "Dutch hospitals" include all medical centers in the Netherlands except ours, "Dutch nursing homes" include all long-term care facilities in the Netherlands, and "other" refers to cases not belonging to one of the above-mentioned healthcare facilities. 


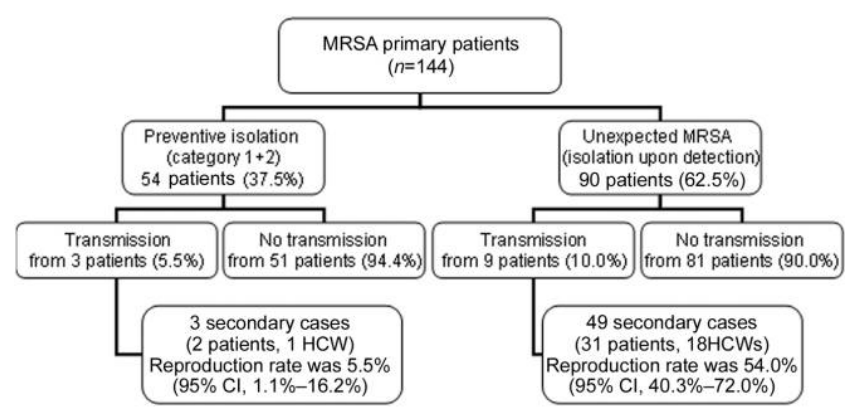

FIGURE 4. Effect of strict isolation on transmission of methicillin-resistant Staphylococcus aureus (MRSA) by primary case patients isolated after detection of MRSA carriage, compared with those preemptively isolated on admission to our hospital in the Netherlands (2000-2004). The reproduction rate of MRSA was statistically significantly lower for primary patients who were preemptively isolated on admission, compared with the reproduction rate for primary patients who were isolated after MRSA was detected $(P<$ $.001)$. A $P$ value of less than .05 was considered to be statistically significant. The $95 \%$ confidence intervals (CIs) and the $P$ values for differences between these groups of primary patients were based on an exact Poisson test.

\section{RES ULT S}

During the 5-year study period, 51,907 MRSA screening cultures were performed for 21,598 persons $(8,403$ patients and 13,195 HCWs). By screening, it was determined that 123 $(1.5 \%)$ of 8,403 patients and $31(0.2 \%)$ of 13,195 HCWs were MRSA carriers. From the performance of clinical cultures, it was determined that 54 additional patients were MRSA carriers, resulting in a total of 177 patients carrying MRSA. The cumulative incidence of MRSA colonization among this group of patients was 0.10 cases per 100 admissions. The cumulative incidence of MRSA colonization (detected by clin- ical culture) among patients who were not at risk (ie, category 3) was 0.03 cases per 100 admissions. The cumulative incidence of MRSA colonization (detected by surveillance culture) among patients at risk (ie, category 2) was 1.46 cases per 100 admissions. Of the 177 patients carrying MRSA, 144 (81\%) were primary cases, and 33 (19\%) secondary cases. The average number of nosocomial transmissions was 6.7 per year. The number of primary cases varied from 2 to 12 cases per quarter of a year (Figure 2). Three patients were known to be MRSA carriers in the past but were thought to be free of MRSA at the time of their readmission. Of the 144 primary cases, 90 had not been isolated until detection of MRSA, which warranted contact screening. Consequently, 142 contact screening rounds were performed in 5 years.

Figure 3 shows the origin of MRSA acquisition for the 144 primary patients and the 12 primary HCWs, resulting in a total of 156 primary cases. Of these 156 cases of MRSA colonization, $44(28 \%)$ were acquired in a foreign healthcare institution, and 45 (29\%) were acquired in other Dutch hospitals, $22(47 \%)$ of which were acquired in a single hospital (in our region) that experienced a major outbreak of MRSA infection in 2002. ${ }^{6}$ There were 16 cases $(10 \%)$ that occurred in a nursing home and another 16 cases (10\%) that fulfilled our definition of community-acquired MRSA colonization; there were 4 cases (3\%) categorized as "other" and 31 cases (20\%) for which the source of MRSA acquisition remained unknown. Of the $31 \mathrm{HCWs}$ who were carriers, $19(61 \%)$ acquired MRSA during patient care in Erasmus MC, 2 (7\%) acquired MRSA by transmission in another Dutch healthcare center, $5(16 \%)$ acquired MRSA in a foreign healthcare institution, and $4(13 \%)$ acquired MRSA in the community; for $1 \mathrm{HCW}(3 \%)$, the source of MRSA acquisition remained unknown.

Figure 4 shows the effect of preemptive isolation on transmission of MRSA. The diversity of PFGE types is given in

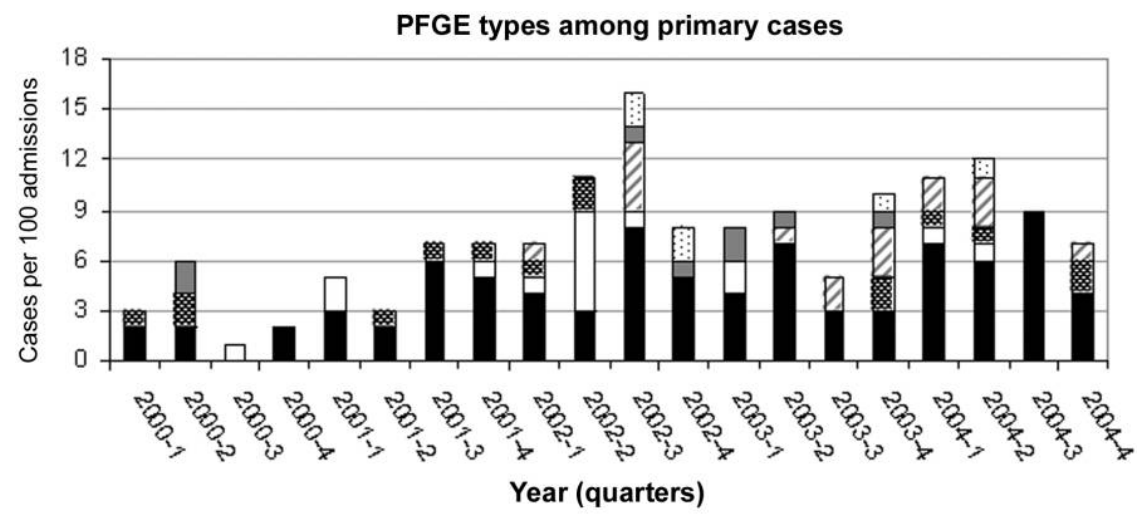

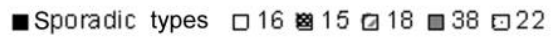

FIGURE 5. Quarterly incidence rates of methicillin-resistant Staphylococcus aureus (MRSA) colonization among primary patients $(n=$ 144) in our hospital in the Netherlands during 2000-2004. Nine strains were lost before typing. Five strains (Dutch pulsed-field type electrophoresis [PFGE] types of PFGE cluster numbers 15, 16, 18, 22, and 38) were more prevalent than other types (which we refer to as sporadic types). 
TA в LE 2. Data on 13 Outbreaks of Methicillin-Resistant Staphylococcus aureus (MRSA) Infection in Erasmus University Medical Center Rotterdam, Rotterdam, the Netherlands

\begin{tabular}{|c|c|c|}
\hline $\begin{array}{l}\text { Year of outbreak, } \\
\text { ward }(s) \text { closed }\end{array}$ & $\begin{array}{c}\text { No. of } \\
\text { days closed }\end{array}$ & $\begin{array}{c}\text { No. of } \\
\text { persons affected }\end{array}$ \\
\hline \multicolumn{3}{|l|}{2000} \\
\hline Urology & 19 & $1 \mathrm{HCW}, 2$ patients \\
\hline \multicolumn{3}{|l|}{2000} \\
\hline Gastroenterology & 28 & 2 HCWs, 7 patients \\
\hline \multicolumn{3}{|l|}{2001} \\
\hline Pediatric ICU & 12 & $2 \mathrm{HCWs}$ \\
\hline \multicolumn{3}{|l|}{2001} \\
\hline ICU & 6 & 3 patients $^{\mathrm{a}}$ \\
\hline \multicolumn{3}{|l|}{2002} \\
\hline General medicine & 19 & $6 \mathrm{HCWs}, 8$ patients \\
\hline \multicolumn{3}{|l|}{$2002^{\mathrm{b}}$} \\
\hline Orthopedics & 21 & 5 HCWs, 1 patient \\
\hline Hemodialysis & 13 & $\ldots$ \\
\hline \multicolumn{3}{|l|}{2002} \\
\hline Cardiosurgery & 20 & $1 \mathrm{HCW}$ \\
\hline \multicolumn{3}{|l|}{2002} \\
\hline Gastroenterology & 16 & 7 patients \\
\hline \multicolumn{3}{|l|}{2002} \\
\hline Gastroenterology & 12 & 1 patient \\
\hline \multicolumn{3}{|l|}{2003} \\
\hline Pediatrics & 11 & 2 patients \\
\hline \multicolumn{3}{|l|}{2003} \\
\hline General medicine ICU & 13 & 1 patient \\
\hline \multicolumn{3}{|l|}{2004} \\
\hline Neurology ICU & 16 & $1 \mathrm{HCW}$ \\
\hline \multicolumn{3}{|l|}{2004} \\
\hline Pediatrics & 14 & $1 \mathrm{HCW}, 1$ patient \\
\hline Total & 220 & 19 HCWs, 33 patients \\
\hline
\end{tabular}

${ }^{a}$ The primary patient died during admission to a single bedroom in the intensive care unit (ICU). He was not known to be infected with MRSA; therefore, his room was not disinfected after his death. However, after his death, clinical culture yielded MRSA. The next patient admitted to the same room was infected by the same strain, which was most likely transmitted from the environment because contact screening of all other patients and healthcare workers (HCWs) on the ICU yielded negative results. This patient was dismissed from the ICU and transmitted MRSA to 2 other patients in another ICU with open ward-style rooms before he was identified as carrying MRSA.

b The same strain was transmitted on 2 different wards. The index patient was a patient who was transferred from another Dutch hospital. At the time of transfer, the patient was not known to be at risk for MRSA infection or colonization and was already transferred to the hemodialysis ward without MRSA precautions before it turned out that he was MRSA positive.

Figure 5. Of the 52 secondary patients, $42(81 \%)$ were infected by 1 of the 5 most prevalent types, $52 \%$ of all strains belong to 1 of those 5 types. In 122 unique strains, staphylococcal cassette chromosome (SCC) mec type was determined. Type I was seen in $22(18 \%)$, type II in $8(7 \%)$, type III in 13 (11\%), and type IV in 42 strains (34\%), and 28 strains (23\%) were untypable.

We had 13 outbreaks of MRSA infection in our hospital, and we were able to define them as being epidemiologically and molecularly linked via the transmission of the pathogen among patients and HCWs (Table 2). Of the 13 outbreaks, 10 were by newly introduced, different strains, and 3 were caused by persons with known MRSA infection or colonization in their history who were thought to be free of MRSA and therefore were not isolated at readmission. Thirteen wards had to be temporarily closed to prevent further MRSA transmission. During the 5-year study period, 5 episodes of MRSA bacteremia occurred in which 4 patients died, an incidence rate of 0.28 cases of infection per 100,000 patientdays per year.

\section{DISCUSSION}

We applied and still apply a strict MRSA search and destroy policy at a large university medical center, and we have succeeded in maintaining a very low incidence of MRSA colonization and infection in the face of increasing rates of colonization and infection in other parts of the world. The use of preemptive isolation decreased the basic reproductive rate of MRSA by nearly 10 -fold. Other Dutch medical centers show comparable results. ${ }^{7}$

Because this was not a randomized controlled study, the precise effect of the search and destroy policy could not be exactly defined but only deduced from years of implementation of the policy, together with years of low incidence of MRSA colonization and infection, which leads us to conclude that this policy is justified and effective. Other countries or hospitals that do not yet have this policy in place but intend to do so should perform such a study. However, the low numbers of nosocomial transmission (ie, secondary cases) is quite likely to be due to the search and destroy policy. The effect of the search and destroy policy should be taken as the effect of a bundle of preventive measures, of which the individual contribution of each measure of the policy cannot be quantified separately. However, we believe that to control the spread of MRSA infection or colonization requires the use of multiple simultaneous interventions, because there are multiple potential sources of MRSA infection or colonization (ie, the patient, the HCWs, and the environment) and multiple routes of transmission. Only by addressing these multiple sources and routes can one maintain or obtain low levels of MRSA endemicity. The MRSA search and destroy policy is a national policy, which had led to the discovery of the observed genetic diversity of MRSA strains in the Netherlands. Because secondary transmission, and thus clonal spread, is not frequent, individual genotypes will not dominate the population of patients with MRSA infection or colonization in the Netherlands. Of the 177 patients who carried MRSA, 85 (48\%) were carriers of MRSA strains with distinct PFGE types that varied, and 92 (52\%) were carriers of 1 of 5 predominant strains; all of these strains were involved in a large outbreak in a nearby hospital. ${ }^{6}$ In 2002 (the period when a large outbreak occurred in a nearby hospital; ie, 1 [20\%] of the 5 years of the study period), 18 (55\%) of the 33 secondary patients and 15 (79\%) of the 19 secondary HCWs were identified. Because of the identification 
of the secondary patients as MRSA carriers and their subsequent isolation on readmission, we only experienced 3 outbreaks related to the recurrence of an already known MRSA genotype.

We only actively screened patients and HCWs at increased risk of carrying MRSA, which we felt was justified because of the low incidence of MRSA carriage among patients at risk and the prevalence of MRSA carriage found in the community in the Netherlands. ${ }^{8}$ Furthermore, we determined that the cumulative incidence of MRSA carriage among patients screened because of an increased risk of MRSA carriage (ie, category 2) was 49 -fold higher than it was among patients not deemed to be at risk. However, the latter incidence data can be an underestimate, because not all patients admitted to the hospital were actively screened or clinically tested for MRSA on culture. As soon as an MRSA carrier is identified, strict isolation procedures are implemented to limit further transmission. Jernigan et $\mathrm{al}^{9}$ estimated the rate of MRSA transmission by a carrier who was not isolated (contact isolation) to be 14 cases per 1,000 patient-days, which is 16 fold higher than the rate of transmission when contact isolation is used. During the study period, we detected 123 (primary and secondary) patients with MRSA carriage who were not isolated for at least part of their stay in the hospital; at the time of detection, $52(42 \%)$ of these 123 patients were considered to be at increased risk of carrying MRSA (ie, category 2). Isolation at admission was associated with a very low MRSA reproductive rate of $5.5 \%$, which is 10 -fold lower than the reproductive rate when isolation is not started at admission. The rate of MRSA transmission among nonisolated patients was much higher than that among isolated patients, but it was limited by the immediate implementation of isolation when MRSA was detected. Bootsma et $\mathrm{al}^{10}$ have calculated in their mathematical model that the isolation of MRSA carriers and the screening and isolation of patients belonging to certain risk categories may successfully decrease a high incidence or retain a low incidence of MRSA infection or colonization. This prediction was confirmed by our observations.

The strategy of preemptive isolation and screening has had a considerable impact on the decrease in days of exposure to MRSA. The proportion of patients detected by screening (123 [69\%] of 177) is comparable to the proportions described elsewhere. ${ }^{11-13}$ Harbarth et $\mathrm{al}^{14}$ found that $55(77 \%)$ of 71 MRSA carriers would have been missed if screening cultures were not performed. Salgado and Farr ${ }^{11}$ found that relying only on clinical microbiological cultures would have failed to identify $85 \%$ of their MRSA-colonized patients. Colonization with methicillin-susceptible $S$. aureus is a risk factor for infections such as bacteremia or surgical site infection. This likely also holds true for colonization with MRSA. ${ }^{15,16} \mathrm{~A}$ low threshold for screening of patients and the use of sensitive laboratory methods ensured that the majority of MRSA-colonized persons were identified and were not just "the tip of the iceberg." The low incidence rate of MRSA bacteremia
(0.28 cases per 100,000 patient-days) is in agreement with the previous statement. In the European Antimicrobial Resistance Surveillance System report of 2004, the incidence rate of MRSA bacteremia in the Netherlands was 0.35 cases per 100,000 patient-days (95\% confidence interval, $0.2-0.7$ cases per 100,000 patient-days). The median incidence rate of MRSA bacteremia in Europe was 3.93 cases per 100,000 patient-days, which is 14 times higher than the median incidence rate of MRSA bacteremia in our center. ${ }^{17}$

Recently, 2 interesting studies were published concerning the fight against MRSA infection or colonization. Harbarth et $\mathrm{al}^{18}$ had to conclude that their universal, rapid MRSA admission screening strategy did not reduce the rate of nosocomial MRSA infection. Robicsek et $\mathrm{al}^{19}$ initiated universal active surveillance and decolonization and reported a $70 \%$ reduction in MRSA disease. However, there were differences between our policy and the policy of Harbarth et al. ${ }^{18}$ In their policy, patients who were admitted to the hospital for less than 24 hours were not screened, even if they were at high risk for MRSA carriage. Also, there was no screening of inpatients to detect MRSA or isolate newly colonized patients, and the screening of HCWs was even not mentioned. ${ }^{20}$ To achieve lower rates of MRSA infection with the use of their policy, the other potential reservoirs of MRSA in hospitals, especially HCWs, will need to be addressed.

Years of use of a strict MRSA search and destroy policy in the Netherlands has coincided with a low prevalence of MRSA infection or colonization. However, Tiemersma et al ${ }^{1}$ reported a significant 3-fold increase in rates of MRSA infection or colonization in the Netherlands in 2002, compared with the years before. An explanation for this increase could be (1) reporting bias, because, at that time, the reference laboratory (ie, the National Institute for Public Health and the Environment) introduced a national PFGE-typing program that directed laboratories to send their strains in, (2) the national introduction of a more sensitive culture method using enrichment broth, ${ }^{4}$ or (3) the large outbreak of MRSA infection that occurred that year in a hospital in our region, with many unidentified carriers being admitted to other medical centers. ${ }^{6,21}$

Threats to the low incidence of MRSA infection or colonization in any hospital are admission of cases of communityacquired MRSA infection and/or cases of MRSA infection resulting from transmission in a nursing home; both types of cases of infection were the sources of MRSA carriage in $10 \%$ of all primary cases of MRSA carriage in our hospital. There was only 1 case of community-acquired MRSA colonization with SCCmec type IV.

The cornerstone of the MRSA search and destroy policy is preventive isolation of high-risk patients before their MRSA carriage is even substantiated. Our results show that one-third of the primary cases acquired their MRSA in a foreign healthcare institution and thus were grouped correctly into 1 of the 2 risk categories. However, 90 (62.5\%) of 144 primary patients were not isolated at the time of 
hospital admission, and $9(10 \%)$ of these 90 patients caused the transmission of MRSA in our medical center. Therefore, research must be continued to identify newly emerging risk factors or to identify groups of patients in the community as belonging to category 2 .

\section{ACKNOWLEDGMENTS}

We thank all infection control practitioners for their hard work and effort isolating patients at risk and performing contact screening.

Potential conflicts of interest. All authors report no conflicts of interest relevant to this article.

Address reprint requests to Margreet C. Vos, MD, PhD, Department of Medical Microbiology and Infectious Diseases, Erasmus University Medical Center Rotterdam, PO Box 2040, 3000 CA Rotterdam, the Netherlands (m.vos@erasmusmc.nl).

Presented in part: 11th International Symposium on Staphylococci and Staphylococcal Infections; Charleston, SC; October 24-27, 2004; and 45th Interscience Conference on Antimicrobial Agents and Chemotherapy; Washington, DC; December 16-19, 2005.

\section{RE F E R E N C E S}

1. Tiemersma EW, Bronzwaer SL, Lyytikainen O, et al. Methicillin-resistant Staphylococcus aureus in Europe, 1999-2002. Emerg Infect Dis 2004; 10: 1627-1634.

2. Dutch Working Party on Infection Prevention (WIP). Policy for methicillin-restistant Staphylococcus aureus, 2003. Available at: http://www.wip .nl. Accessed January 2000.

3. Goossens H, Ferech M, Vander Stichele R, Elseviers M. Outpatient antibiotic use in Europe and association with resistance: a cross-national database study. Lancet 2005; 365:579-587.

4. Wertheim H, Verbrugh HA, van Pelt C, de Man P, van Belkum A, Vos MC. Improved detection of methicillin-resistant Staphylococcus aureus using phenyl mannitol broth containing aztreonam and ceftizoxime. J Clin Microbiol 2001; 39:2660-2662.

5. Kerremans JJ, Maaskant J, Verbrugh HA, van Leeuwen WB, Vos MC Detection of methicillin-resistant Staphylococcus aureus in a low-prevalence setting by polymerase chain reaction with a selective enrichment broth. Diagn Microbiol Infect Dis 2008; 61:396-401.

6. van Trijp MJCA, Melles DC, Hendriks WDH, Parlevliet GA, Gommans M, Ott A. Successful control of widespread methicillin-resistant Staphylococcus aureus colonization and infection in a large teaching hospital in the Netherlands. Infect Control Hosp Epidemiol 2007; 28:970-975.

7. Vriens M, Blok H, Fluit A, Troelstra A, van Der Werken C, Verhoef J.
Costs associated with a strict policy to eradicate methicillin-resistant Staphylococcus aureus in a Dutch University Medical Center: a 10-year survey. Eur J Clin Microbiol Infect Dis 2002; 21:782-786.

8. Wertheim HF, Vos MC, Boelens HA, et al. Low prevalence of methicillinresistant Staphylococcus aureus (MRSA) at hospital admission in the Netherlands: the value of search and destroy and restrictive antibiotic use. $J$ Hosp Infect 2004; 56:321-325.

9. Jernigan JA, Titus MG, Groschel DH, Getchell-White S, Farr BM. Effectiveness of contact isolation during a hospital outbreak of methicillinresistant Staphylococcus aureus. Am J Epidemiol 1996; 143:496-504.

10. Bootsma MC, Diekmann O, Bonten MJ. Controlling methicillin-resistant Staphylococcus aureus: quantifying the effects of interventions and rapid diagnostic testing. Proc Natl Acad Sci U S A 2006; 103:5620-5625.

11. Salgado CD, Farr BM. What proportion of hospital patients colonized with methicillin-resistant Staphylococcus aureus are identified by clinical microbiological cultures? Infect Control Hosp Epidemiol 2006; 27:116-121.

12. Lucet JC, Grenet K, Armand-Lefevre L, et al. High prevalence of carriage of methicillin-resistant Staphylococcus aureus at hospital admission in elderly patients: implications for infection control strategies. Infect Control Hosp Epidemiol 2005; 26:121-126.

13. Girou E, Azar J, Wolkenstein P, Cizeau F, Brun-Buisson C, Roujeau JC. Comparison of systematic versus selective screening for methicillin-resistant Staphylococcus aureus carriage in a high-risk dermatology ward. Infect Control Hosp Epidemiol 2000; 21:583-587.

14. Harbarth S, Masuet-Aumatell C, Schrenzel J, et al. Evaluation of rapid screening and pre-emptive contact isolation for detecting and controlling methicillin-resistant Staphylococcus aureus in critical care: an interventional cohort study. Crit Care 2006; 10:R25.

15. von Eiff C, Becker K, Machka K, Stammer H, Peters G. Nasal carriage as a source of Staphylococcus aureus bacteremia. N Engl J Med 2001; 344: 11-16.

16. Kluytmans JA, Mouton JW, Ijzerman EP, et al. Nasal carriage of Staphylococcus aureus as a major risk factor for wound infections after cardiac surgery. J Infect Dis 1995; 171:216-219.

17. The European Antimicrobial Resistance Surveillance System (EARSS). Annual Report 2004. Available at: http://www.earss.rivm.nl. Accessed November 2008.

18. Harbarth S, Fankhauser C, Schrenzel J, et al. Universal screening for methicillin-resistant Staphylococcus aureus at hospital admission and nosocomial infection in surgical patients. JAMA 2008; 299:1149-1157.

19. Robicsek A, Beaumont JL, Paule SM, et al. Universal surveillance for methicillin-resistant Staphylococcus aureus in 3 affiliated hospitals. Ann Intern Med 2008; 148:409-418.

20. Salgado CD, Vos MC, Farr BM. Universal screening for methicillin-resistant Staphylococcus aureus by hospitals. JAMA 2008; 300:503-504.

21. Vos MC, Ott A, Verbrugh HA. Successful search-and-destroy policy for methicillin-resistant Staphylococcus aureus in the Netherlands. J Clin Microbiol 2005; 43:2034-2035. 